\title{
Auf dem Weg zu mehr Arbeitsteilung durch Open-Source?
}

\author{
Eine fallstudienbasierte Untersuchung \\ am Beispiel von db4objects
}

\author{
Christian M. Wolf', Philipp Nguyen Khuong², Alexander Benlian', \\ Thomas Hess ${ }^{1}$ \\ ${ }^{1}$ Institut für Wirtschaftsinformatik. und Neue Medien, \\ Ludwig-Maximilians-Universität München \\ ${ }^{2} V$ ersant Corporation (db4o)
}

\section{Einleitung}

Die Entwicklung der Wertschöpfungsstruktur des Automobilsektors stellt unbestritten ein Musterbeispiel für die zunehmende Arbeitsteilung innerhalb einer gesamten Industrie dar. Diese Entwicklung resultiert bekanntermaßen in einer zunehmenden vertikalen Desintegration der eigentlichen Fahrzeughersteller, deren Leistungstiefe bei den deutschen Unternehmen beispielsweise nur noch bei zehn bis $25 \%$ liegt und weiterhin eine fallende Tendenz aufweist (Kretschmer 2005, S. 82). Die von den Fahrzeugherstellern abgegebenen Wertschöpfungsanteile werden dabei insbesondere von vorgelagerten und eng kooperierenden Zulieferern übernommen. Ähnlich verhält es sich in der chemischen Industrie: Auch hier desintegrieren die großen Player und produzieren zunehmend lediglich chemische Grundprodukte, die dann - im Gegensatz zum zuvor erwähnten Automobilsektor - von nachgelagerten spezialisierten Unternehmen veredelt werden.

Aus der Branchenperspektive prognostizieren transaktionskostenbasierte Analysen für die Software-Industrie zum einen eine Fragmentierung der Wertschöpfung (,Move-to-the-market"). Zum anderen lassen sich in der Software-Industrie empirisch aber auch spezifische Investitionen in eine geringe Anzahl von Partnern feststellen, die aus Transaktionskostensicht zur Prognose von Kooperationsstrategien führen („Move-to-the-middle“) (Buxmann et al. 2008, S. 57).

Im Rahmen einer betriebswirtschaftlichen und somit unternehmensbezogenen Analyse stellt sich für Softwareunternehmen daher die Frage, ob eine ähnliche zunehmende Arbeitsteilung wie bei Kraftfahrzeugherstellern oder Chemieunternehmen festzustellen ist. Dieser Fragenkomplex ist gleichbedeutend mit dem Themen- 
feld des Sourcings eigener Aufgaben eines Softwareunternehmens, welches im Schrifttum bisher noch nicht hinlänglich bearbeitet wurde und die Frage nach den effizienten Grenzen und somit der Leistungstiefe eines Softwareunternehmens aufwirft (Buxmann et al. 2008, S. 51 u. 55).

Zwar findet sich im generellen Themenfeld der Leistungstiefe eine große Vielzahl an Arbeiten, die sich mit Unternehmen aus nahezu allen denkbaren Branchen beschäftigen - Untersuchungen bei Softwareunternehmen finden sich jedoch bisher kaum. So konnten beispielsweise Wolf et al. (2008) für den Fall eines Softwareunternehmens mit klassischem Closed-Source-Geschäftsmodell (SAP) eine zunehmende zwischenbetriebliche Arbeitsteilung mit der Folge einer sinkenden Leistungstiefe nachweisen. Auf Grund der offenen Struktur von Open-SourceSoftware und des dazugehörigen Geschäftsmodells liegt die Vermutung nahe, dass die Arbeitsteilung in diesem Bereich noch deutlich stärker ausgeprägt ist als im klassischen Closed-Source-Geschäftsmodell (Sharma et al. 2002, S. 10). Um einen weiteren Beitrag zur Forschung der Wirtschaftsinformatik im Bereich der Software-Industrie hinsichtlich der Ausdifferenzierung der Wertschöpfung zu leisten, untersuchten wir daher mittels einer Fallstudie des Unternehmens db4obejcts, ob und in welchen Bereichen der Wertschöpfungskette auch bei einem typischen Softwareunternehmen mit Open-Source-Geschäftsmodell eine zunehmende unternehmensexterne Arbeitsteilung feststellbar ist. Mit dem Ziel festzustellen, ob sich Softwareunternehmen auf dem Weg zu mehr Arbeitsteilung durch OpenSource befinden, versuchten wir daher folgende Forschungsfragen zu beantworten: Wie ist die unternehmensexterne Arbeitsteilung aus der Sicht von db4objects aktuell ausgeprägt, wie war sie bei der Gründung des Unternehmens ausgestaltet und bei welchen Wertschöpfungsaktivitäten haben sich Veränderungen ergeben? Zur Beantwortung erhoben wir für zwei verschiedene Betrachtungszeitpunkte, welche Wertschöpfungsaktivitäten vom Unternehmen selbst durchgeführt, welche von kooperierenden Partnern erbracht und welche über den Markt bezogen wurden, um dann anschließend vergleichen zu können, wie sich die daraus resultierende Leistungstiefe entwickelt hat.

In Kapitel 2 erfolgt zunächst eine theoretische Einordnung der unternehmensexternen Arbeitsteilung und der daraus resultierenden Leistungstiefe. Im 3. Kapitel stellen wir die Konzeption unserer Fallstudienuntersuchung vor, deren Ergebnisse Bestandteil des 4. Kapitels sind. In Kapitel 5 schließen wir mit einem Fazit sowie einem Ausblick auf weiteren Forschungsbedarf.

\section{Unternehmensexterne Arbeitsteilung und Leistungstiefe}

Der Begriff der Arbeitsteilung wird im Schrifttum in eine horizontale und eine vertikale Variante eingeteilt. Werden Aufgaben auf mehrere organisatorische Einheiten verteilt, wobei jede Einheit dieselbe Teilaufgabe erfüllt, liegt horizontale Arbeitsteilung vor. Wird eine Gesamtaufgabe hingegen in Teilaufgaben unterschiedli- 
cher Art zerlegt, die dann von verschiedenen spezialisierten Akteuren wahrgenommen werden, spricht man von vertikaler Arbeitsteilung. Nur die vertikale Arbeitsteilung führt demnach zu inhaltlichen Merkmalsunterschieden der zu erfüllenden Teilaufgaben und somit zu einer Spezialisierung der beteiligten Akteure (Zäpfel 1989, S. 157). Des Weiteren kann in innerbetriebliche und zwischenbetriebliche Arbeitsteilung unterschieden werden. Erstere bedeutet, dass Teilaufgaben in spezialisierten Organisationseinheiten innerhalb eines Unternehmens (z. B. Stellen, Abteilungen) gebündelt werden. Zweite bedeutet hingegen, dass Teilaufgaben in spezialisierten Unternehmen gebündelt werden. Um der Tatsache Rechnung zu tragen, dass im Spezialfall von Open-Source-Software Teilaufgaben auch von kommerziellen wie privaten Nutzern übernommen werden, sprechen wir in diesem Zusammenhang von unternehmensexterner Arbeitsteilung anstatt von zwischenbetrieblicher Arbeitsteilung.

In unserer Untersuchung erfolgte eine Fokussierung auf die unternehmensexterne vertikale Arbeitsteilung. Diese kann zur Folge haben, dass ein Unternehmen nicht mehr sämtliche Aktivitäten für ein Endprodukt innerhalb seiner eigenen Unternehmensgrenzen erbringt. Der Umfang an Wertschöpfung, welchen ein Unternehmen durch seinen eigenen Beitrag im Verhältnis zur insgesamt erforderlichen Wertschöpfung für ein Endprodukt selbst durchführt, wird dabei durch den Begriff der Leistungstiefe charakterisiert (Zäpfel 1989, S. 132). Synonym zur Leistungstiefe ist der Begriff der vertikalen Integration (Picot 1991, S. 337).

Vertikale Integrationsentscheidungen sind dabei nicht nur auf Fremd- oder Eigenbezugs- bzw. Eigenabsatzentscheidungen (sog. Make-or-Buy-Entscheidungen) beschränkt, sondern beinhalten auch verschiedenste Formen von Unternehmenskooperationen. Die unterschiedlichen Ausprägungen der so möglichen Koordinationsformen können in der Theorie nicht vollständig abgebildet werden, was laut Fauser (2004, S. 12) ein Grundproblem der Organisationsforschung darstellt. Williamson hat zur Überwindung dieser Problematik die zwei sich in einem antagonistischen Verhältnis gegenüberstehenden Idealtypen des Markts und der Hierarchie eingeführt (Fauser 2004, S. 12; Williamson 1991, S. 280; Williamson 2002, S. 180182): Kennzeichen des Markts ist die punktuelle Zusammenarbeit und Koordination über den Preismechanismus. In Unternehmen herrscht hingegen die auf längerfristiger vertraglicher Zusammenarbeit basierende hierarchische Koordination vor. Daher wird die Verschiebung einer Aktivität von der hierarchischen Koordination zum Markt als vertikale Desintegration bezeichnet. Zwischen den genannten Extremen Hierarchie und Markt existiert eine Vielfalt an als hybride Koordinationsformen bezeichneten und durch ihre Integrationsintensität unterscheidbaren, institutionellen Arrangements. Williamson fasst diese Vielfalt zur Komplexitätsreduktion als „Hybride“ zusammen, sodass sich letztlich die drei Idealtypen Markt, Hybrid und Hierarchie ergeben (Fauser 2004, S. 12; Williamson 1991, S. 269). Auch in unserer Untersuchung erfolgte eine Reduktion auf die drei vorgestellten Idealtypen anstatt einer Betrachtung des gesamten Spektrums möglicher Koordinationsformen. Wie Fauser (2004, S. 12-13) feststellt, birgt die Verwendung von Idealtypen 
dabei die folgenden Vor- und Nachteile: Die Vorteile liegen in der deutlichen Komplexitätsreduktion, der Erhöhung der Transparenz der Analysen sowie der Ableitung von zwar weniger differenzierten, dafür aber eindeutigeren und damit aussagekräftigeren Ergebnissen. Nachteile sind die mit Idealtypen verbundene stark vereinfachte Darstellung der Realität, sodass die gewonnenen Erkenntnisse mehr den Charakter von Tendenzaussagen haben (Laux und Liermann 1993, S. 2829), die eindimensionale Betrachtungsweise (Richter 2003, S. 13), sowie die mögliche verstärkte Polarisierung abgeleiteter Aussagen (Remer 1996, S. 107). Zusammenfassend stellt diese Vorgehensweise also eine deutliche Realitätsvereinfachung dar - die zur Anwendung kommenden konstruierten Idealtypen ermöglichen es jedoch, zu deutlich eindeutigeren Ergebnissen zu kommen, sodass die Vorteile der Komplexitätsreduktion die Nachteile der Realitätsvereinfachung kompensieren (Fauser 2004, S. 12-13; Remer 1996, S. 105).

\section{Konzeption der Fallstudienuntersuchung}

Das Fallstudienobjekt der vorliegenden Untersuchung ist das Unternehmen db4objects (siehe auch www.db4objects.com), welches kurz nach Abschluss unserer Untersuchung im Dezember 2008 von Versant übernommen wurde. db4objects zeichnet sich für die Entwicklung und kommerzielle Lizensierung der OpenSource-Objektdatenbank „database for objects“ (db4o) verantwortlich. Das OpenSource-typische Geschäftsmodell von db4o basiert auf einer dualen Lizenz. Dies bedeutet, dass die Software entweder unter einer kommerziellen Lizenz oder unter bestimmten Bedingungen kostenlos unter der GNU General Public License (GPL) lizensiert werden kann. Eine duale Lizenz ist für Anwender insbesondere im Hinblick auf die Kosten interessant. So können nicht-kommerzielle Nutzer wie Bildungseinrichtungen sowie Personen oder Unternehmen, welche db4o nur intern nutzen und nicht als Teil ihrer Applikation weiterdistribuieren, kostenlos auf die GPL-Variante zurückgreifen. Demgegenüber wird für die kommerzielle Nutzung ein volumenbasiertes Lizenz- und Preismodell angeboten.

Die gesamte Fallstudie orientierte sich hinsichtlich Design, Ablauf und Auswertung an dem Konzept und den Empfehlungen von Yin (2003). Dem Gütekriterium der Repräsentativität wurde im Rahmen der Möglichkeiten einer explorativen Einzelfallstudie durch die bewusste und gezielte Auswahl von db4obejcts als Fallstudienobjekt Rechnung getragen, da das Unternehmen als typischer Fall für ein Open-Source-Geschäftsmodell angesehen werden kann (Meyer 2003, S. 477). Zur Sicherung der Reliabilität liefern wir im Folgenden eine akribische Dokumentation des Forschungsprozesses, die Transparenz bezüglich des Ablaufs der Untersuchung schafft und somit ihre Wiederhol- bzw. Überprüfbarkeit gewährleistet (Yin 2003, S. 34-39). Die Datenerhebung erfolgte in einem in Abbildung 1 dargestellten Dreiphasenmodell. 

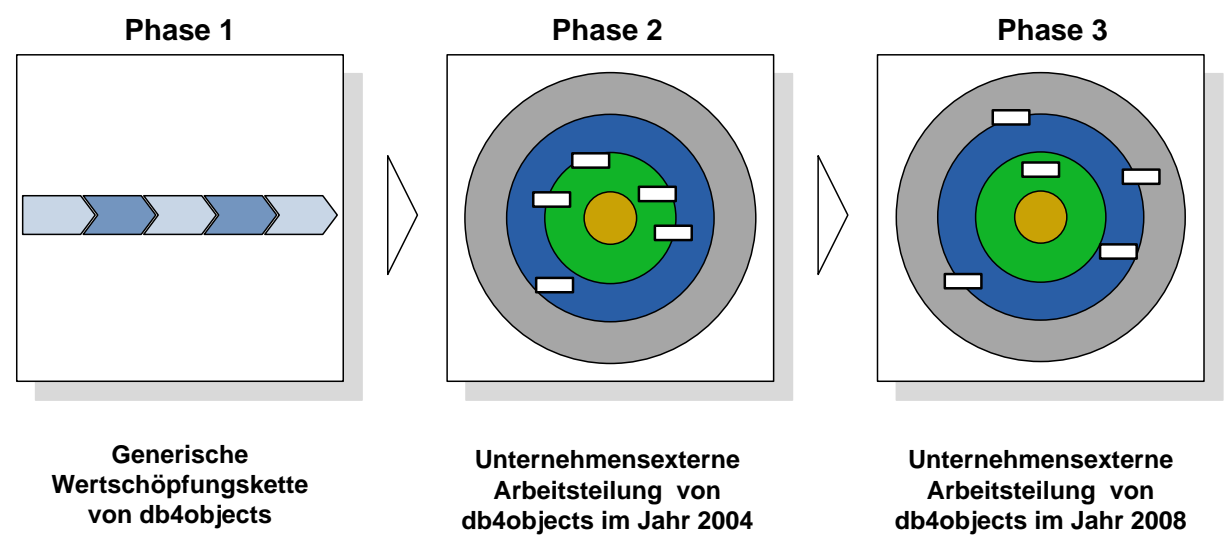

\section{Abbildung 1: Dreiphasenmodell der Datenerhebung}

Der in den Erhebungsphasen 2 und 3 sowie bei der in Kapitel 4 folgenden Auswertung zur Anwendung gekommene Untersuchungsrahmen ist stark an die Visualisierung von Business Webs nach Franz (2003) angelehnt. Zwar zielen Business Webs nicht auf die Klassifikation der unternehmensexternen Arbeitsteilung bei der Erfüllung einzelner Wertschöpfungsaktivitäten $\mathrm{ab}$, sondern analysieren konkrete Unternehmen und ihre Beziehungen. Ihre grundsätzliche Darstellungsform in kreisförmigen Schichten erscheint jedoch sehr geeignet, um den Grad an unternehmensexterner Arbeitsteilung bei der Erfüllung einer Wertschöpfungsaktivität zu klassifizieren und eingängig zu visualisieren: Die Wertschöpfungsaktivitäten werden dabei nach dem Grad der unternehmensexternen Arbeitsteilung von „db4objects“ über „Partner“ bis „Markt“ eingeteilt und von innen nach außen um den im Zentrum der Wertschöpfung stehenden Kunden herum positioniert, wobei diese Positionierung ordinalskaliert ist. Das bedeutet, je weiter innen eine Wertschöpfungsaktivität verortet werden kann, desto geringer ist der Grad an unternehmensexterner Arbeitsteilung aus der Sicht des fokalen Unternehmens db4objects. Dabei sind Mischformen zwischen Kategorien möglich, wenn Wertschöpfungsaktivitäten oder Teile davon beispielsweise sowohl von db4objects als auch von Partnern durchgeführt werden.

Leitfadengestützte Experteninterviews bildeten den Ausgangs- und Mittelpunkt der Erhebung, da nur eine eingeschränkte Anzahl von themenrelevanten Dokumenten zur Verfügung stand. Die Interviews waren teilstandardisiert, d. h. es wurde auf einen Fragenkatalog und Gesprächsleitfaden zurückgegriffen, um eine Struktur in die Gespräche zu bringen. Obwohl alle notwendigen Daten durch Interviews erhoben werden konnten, wurde zur Sicherung der Validität der Untersuchung, wie von Yin (2003, S. 98-99) empfohlen, im Sinne einer Datentriangulation auf eine ergänzende Dokumentenanalyse zurückgegriffen und die Ergebnisse wurden durch die wichtigsten Informanden durchgesehen. Als Datenquellen der Dokumentenanalyse dienten Newsletter, Pressemitteilungen, offizielle Blogeinträge 
sowie frühere Interviews, welche auf der offiziellen Firmen-Homepage und im Internet einzusehen sind.

Ziel von Phase 1 war es, zunächst die genaue Wertschöpfungskette der Erstellung und Verwertung des Produkts db4o zu erheben, um danach die unternehmensexterne Arbeitsteilung der identifizierten Teilaktivitäten im Jahre 2004 und 2008 sowie Gründe für einen etwaigen Wandel dokumentieren zu können. Zu diesem Zweck wurden der Chief Executive Officer (CEO) und der Chief Software Architect (CSA) von db4objects befragt. Vor der eigentlichen Erhebung wurde den Interviewpartnern ein Einführungsdokument in unsere Studie vorgelegt, um ihnen eine grundlegende Vorbereitung zu ermöglichen. Als nächster Schritt wurde den Befragten dann ein auf einer generischen und damit allgemeingültigen Wertschöpfungskette von Softwareunternehmen basierender Entwurf der konkreten db4oWertschöpfungskette vorgelegt, mit der Bitte, diesen unabhängig voneinander nach bestem Wissen zu komplettieren und eventuelle Fehler zu korrigieren. Als nächster Schritt wurden zwei voneinander getrennte, etwa 30 Minuten dauernde Interviews durchgeführt, in denen von den Befragten alle Teilaktivitäten im Zusammenhang mit der Wertschöpfung erklärt und durchgesprochen wurden. Als letzter Schritt der ersten Erhebungsphase wurde beiden Interviewteilnehmern eine finale Version der db4o-Wertschöpfungskette vorgelegt, um anschließend in einer gemeinsamen Konferenzschaltung letzte Detailkorrekturen vorzunehmen. So wurden bspw. auf einigen Wertschöpfungsstufen sehr granulare Unteraktivitäten bestimmter Wertschöpfungsschritte in einem Oberpunkt aggregiert, um die Komplexität der nachfolgenden Erhebungen nicht unnötig zu erhöhen. Im Zuge dieser letzten Korrekturen erfolgte auch die gemeinsame Validierung der Wertschöpfungskette durch beide Befragten.

Anschließend wurde in Phase 2 im Rahmen einer Vergangenheitsbetrachtung die unternehmensexterne Arbeitsteilung im Gründungsjahr 2004 erhoben. Dazu wurden insgesamt drei Personen befragt (CEO, CSA und Chief Financial Officer). In den jeweils etwa 30 Minuten dauernden Einzelinterviews kamen Checklisten zum Einsatz. Den Befragten wurde dabei eine auf den Ergebnissen aus Phase 1 aufbauende Übersicht mit allen Wertschöpfungsaktivitäten vorgelegt. Zusammen mit den Interviewpartnern wurden die einzelnen Wertschöpfungsaktivitäten Schritt für Schritt im Bezug auf die unternehmensexterne Arbeitsteilung bei der Leistungserbringung abgeprüft. Zum Abschluss wurde das Ergebnis der Erhebung in Form einer Übersicht mit den jeweiligen Koordinationsformen der Wertschöpfungsaktivitäten im Jahr 2004 zur Validierung präsentiert.

Phase 3 folgte dem Vorgehen aus Phase 2 um die unternehmensexterne Arbeitsteilung im Jahr 2008 zu erheben. Dabei wurde zusätzlich eine vierte Person, der Vice President of Engineering des Unternehmens, befragt. Nach Abschluss der Auswertung von Phase 2 und 3 wurde allen Befragten eine Übersicht vorgelegt, welche die Unterschiede und Veränderungen der unternehmensexternen Arbeitsteilung in den Jahren 2004 und 2008 dokumentierte und gleichzeitig als Validierungsgrundlage diente. Dieser Schritt stellte den Abschluss der Datenerhebung 
durch Interviews dar. Die Dokumentenanalyse wurde dabei während des gesamten Erhebungszeitraums kontinuierlich als unterstützender und zusätzlich validierender Prozess durchgeführt.

\section{Ergebnisse der Fallstudienuntersuchung}

Im Jahr 2004 dominierten hierarchische Wertschöpfungsarrangements die Wertschöpfungsstruktur von db4objects. Die daraus resultierende hohe Leistungstiefe des Unternehmens ist auf die damals noch geringe Bekanntheit und somit kleine Nutzer-Community des Produkts zurückzuführen. Dieser Zustand brachte mit sich, dass es noch keine geeigneten Kooperationspartner im Sinne von db4o-Nutzern mit Expertise gab, an die Wertschöpfungsteilaktivitäten insbesondere im Bereich der Produktentwicklung ausgelagert werden konnten. Dennoch waren auf den Wertschöpfungsstufen Marketing und Distribution schon im Gründungsjahr 2004 auch Kooperationen und marktliche Koordination anzutreffen. Auf der Wertschöpfungsstufe Marketing wurde über „Community-Seiten“ mit den Nutzern kooperiert. Hier liegt es in der Natur des Open-Source-Geschäftsmodells, mit den Nutzern über eine Plattform zu kooperieren, auf der sich diese untereinander und mit dem Unternehmen selbst austauschen sowie nutzergenerierte Inhalte in Form von eigenen Projekten und anderen Beiträgen bereitstellen können. Die Wertschöpfungsteilaktivität „Pay-per-Click“-Kampagnen war an eine spezialisierte Internetmarketing-Agentur ausgelagert, die gleichzeitig auch die Teilaktivität der Suchmaschinenoptimierung des Internetauftritts übernahm. Ebenso waren alle Leistungen der Teilaktivität „Public Relations“ über den freien Markt an einen weiteren Dienstleister vergeben. db4objects gab seine Pressemitteilungen und Fachartikel lediglich zur Distribution an den Dienstleister weiter, ohne vorher entschieden zu haben, wo diese platziert bzw. veröffentlicht werden sollten. Ebenso betreute dieser Dienstleister alle Autoren, die Artikel über db4o verfassten oder verfassen wollten. Auf der Wertschöpfungsstufe Distribution wurde im Bereich der Teilaktivität „Internet Distribution“ kooperiert. Obwohl die Distribution von Software über das Internet mit sehr geringen Kosten verbunden ist, wurde hierbei insbesondere in Asien und Lateinamerika mit Partnern zusammengearbeitet, welche db4o auf den unterschiedlichen Portalen ihres jeweiligen Landes zum Download bereitstellten und mit redaktionellen Inhalten anreicherten.

Im Vergleich zu 2004 hatte die Leistungstiefe von db4objects 2008 deutlich abgenommen und zahlreiche Wertschöpfungsteilaktivitäten wurden nun in Kooperation erbracht. Auf der Wertschöpfungsstufe Entwicklung wurden zwar weiterhin einige Teilaktivitäten hierarchisch koordiniert, allerdings gleichzeitig weitaus mehr in Kooperation mit der Nutzer-Community durchgeführt oder über den freien Markt bezogen. So hatten die Nutzer nun Einfluss auf die „Roadmap“ und das „Design“ des Produkts. Die Roadmap konnte direkt über Abstimmungen beeinflusst werden, wohingegen auf das Design nur indirekt über die Implementierung 
der von den Nutzern gewünschten Features Einfluss genommen wurde. Die Entwicklung des Kernprodukts erfolgte zwar nach wie vor hierarchisch, allerdings wurden alle Plugins und Addons entweder in Kooperation mit Nutzern erstellt oder über den Markt bezogen. Im Falle der Kooperation gab es hierzu zum einen von db4objects initiierte Ausschreibungen und Wettbewerbe und zum anderen erstellten Nutzer auch in Eigeninitiative zusätzliche Komplemente. Auch beim Qualitätsmanagement und der Wartung des Kernprodukts wurde eng mit der NutzerCommunity kooperiert. Zum Zwecke des Qualitätsmanagements wurde ein öffentliches Tracking-System eingeführt, welches einen detaillierten Überblick über alle Informationen im Zusammenhang mit den Fehlern im Programmcode gab. Die Nutzer konnten darüber hinaus eigene Test-Cases übermitteln bzw. das Ergebnis ihrer Tests veröffentlichen. Im Rahmen der Wartung des Kernprodukts konnten die Nutzer in so genannten Performance-Contests eigenen, verbesserten Programmcode an db4objects schicken. Die Dokumentation wurde von Nutzern aus der Community, teilweise gegen Entlohnung und teilweise auf freiwiller Basis, in verschiedenste Sprachen übersetzt. Auch auf der Wertschöpfungsstufe Marketing wurden 2008 mehr Teilaktivitäten als 2004 in Kooperation erbracht. Neben dem Produktmanagement betraf dies vor allem so genannte virale Marketingaktivitäten wie Blogeinträge und Beiträge auf anderen Homepages, die von Nutzern verfasst wurden. Des Weiteren wurden Events, wie z. B. Fachkonferenzen, in Kooperation mit „Heavy Usern“ aus der Community organisiert. Darüber hinaus organisierte die Community teils eigenständig, teils in Absprache mit db4objects kleinere, lokale Nutzertreffen. Außerdem wurde einigen erfahrenen Nutzern die Möglichkeit eröffnet, gegen Erstattung der Reisekosten als Speaker im Namen von db4objects an Konferenzen teilzunehmen. Die Aktivitäten im Rahmen der Public Relations wurden hingegen im Gegensatz zu 2004 wieder ingesourct. Während die Wertschöpfungsstufe Vertrieb \& Distribution auch 2008 rein intern durchgeführt wurde, gab es auf der Wertschöpfungsstufe Support \& Consulting nun auch Wertschöpfungsteilaktivitäten, die in Kooperation mit der Nutzer-Community durchgeführt wurden. So leisteten Nutzer in den Community-Foren kostenlosen Presales-Support für andere Nutzer. Das Consulting vor Ort wurde 2008 nicht mehr nur von db4objects alleine, sondern in einigen Ländern in Kooperation mit Heavy Usern durchgeführt.

In Abbildung 2 sind die Wertschöpfungsstufen (WS-Stufen) von db4objects sowie die erhobenen Ausprägungen der Koordinationsformen aller Wertschöpfungsteilaktivitäten im Vergleich zwischen 2004 und 2008 dargestellt. Der Buchstabe „H“ steht dabei für Hierarchie, „, $\mathrm{K}$ “ für hybride Kooperation und „M“ für Markt.

Vergleicht man die Koordinationsformen der Wertschöpfungsaktivitäten von 2004 und 2008 ist auffallend, dass db4objects, gemessen an der Anzahl der Wertschöpfungsaktivitäten im Jahr 2004, eine sehr hohe Leistungstiefe aufwies. Fast alle Aktivitäten wurden intern im Unternehmen durchgeführt - lediglich im Bereich des Marketing und der Distribution wurden Leistungen in Kooperation erbracht oder vom Markt bezogen. 


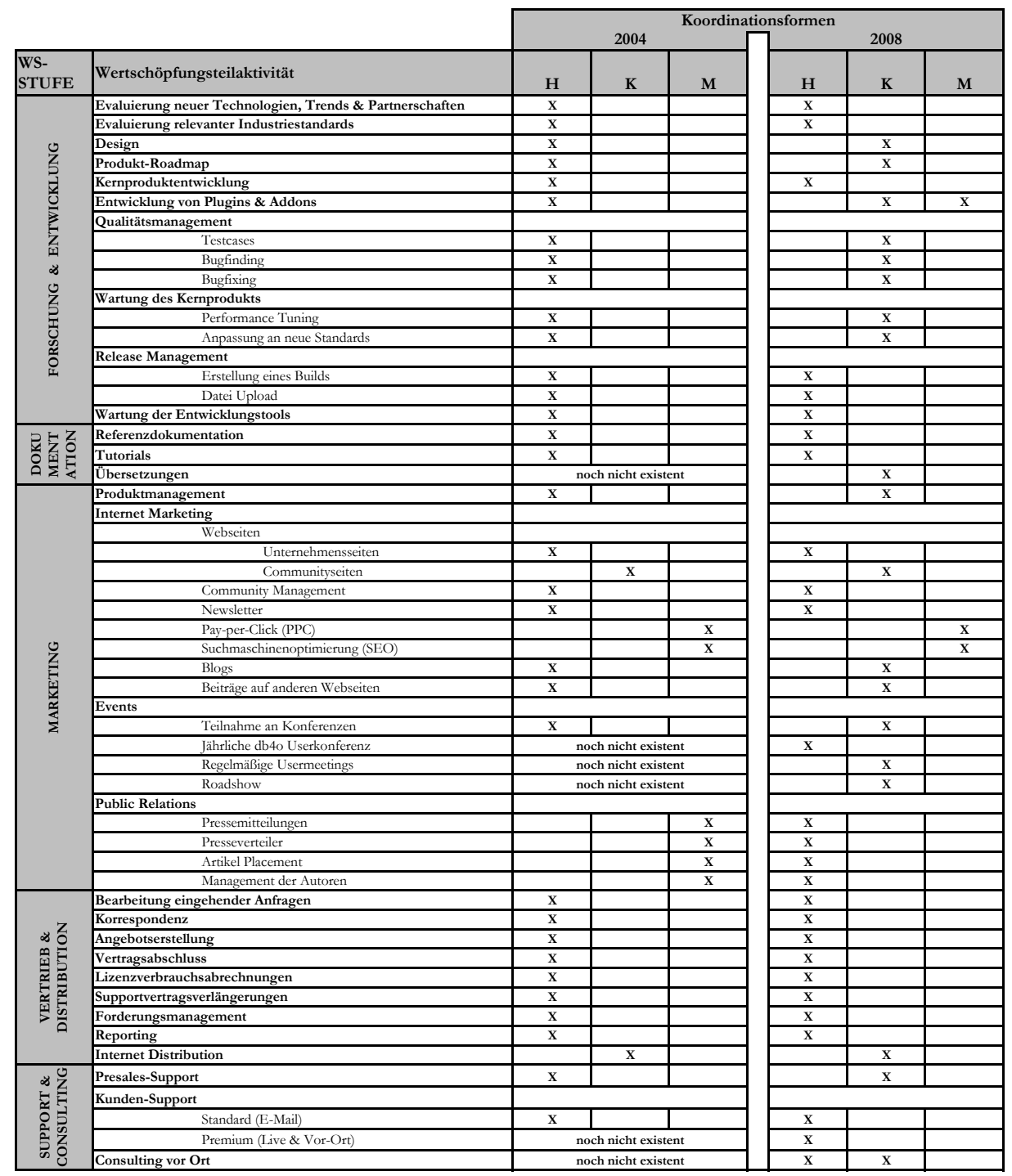

Abbildung 2: Koordinationsformen der Wertschöpfungsaktivitäten im Vergleich

Demgegenüber wurden 2008 erheblich mehr Wertschöpfungsteilaktivitäten in Kooperation mit der Nutzer-Community erbracht sowie weitere Leistungen vom Markt bezogen - die Leistungstiefe hat demnach deutlich abgenommen. Am stärksten ist die Veränderung im Bereich der Entwicklung. Hier wurde bei zahlreichen Aktivitäten von der rein hierarchischen Koordinationsform zur Kooperation übergegangen. Darüber hinaus wurde bei der Entwicklung von Plugins und Addons von der Hierarchie sowohl zur Kooperation als auch zum Marktbezug gewechselt. Weitere größere Änderungen haben sich beim Marketing ergeben, 
auch hier gab es einen Trend hin zur Kooperation. Doch es gab auch eine Änderung der Wertschöpfungsarrangements in die entgegengesetzte Richtung. So wurden alle Aktivitäten der Public Relations im Jahr 2008 wieder hierarchisch koordiniert, nachdem sie in der Vergangenheit an den Markt ausgelagert waren.

Der ausschlaggebende Grund für die abgenommene Leistungstiefe in den genannten Bereichen sowie die dort neu hinzugekommenen Aktivitäten war die stark gewachsene Nutzer-Community. Durch den Anstieg der Nutzeranzahl sowie der Nutzer-Expertise konnte die Community im Zeitablauf zunehmend an den einzelnen Aktivitäten beteiligt werden, um von den Vorteilen des Open-Source-Modells zu profitieren. Hierzu zählen im Vergleich zu traditionellen Softwareprodukten beispielsweise die breitere Entwicklerbasis, der höhere Nutzer-Input und die geringeren Marketingkosten (Sharma et al. 2002, S. 8). Diese für die Open-SourceBranche typische Arbeitsteilung zwischen Herstellerunternehmen und Kunden wird auch interaktive Wertschöpfung genannt (Reichwald und Piller 2006). Es gab jedoch auch Entscheidungen, die ohne Bezug zum Open-Source-Modell rein aus Kostengründen getroffen wurden. Dazu zählen die Wiedereingliederung der Public-Relations-Aktivitäten sowie die Auslagerung einer Plugin-Entwicklung an einen indischen IT-Dienstleister.

Die nachfolgende Abbildung 3 fasst die zuvor erläuterten Veränderungen der unternehmensexternen Arbeitsteilung in der Darstellungsform unseres an Business Webs angelehnten und in Kapitel 4 beschriebenen Untersuchungsrahmens nochmals grafisch zusammen. Wertschöpfungsteilaktivitäten, bei denen keine Veränderungen festgestellt werden konnten, sind aus Gründen der Übersichtlichkeit nicht aufgeführt. Die transparenten Rechtecke stellen dabei die Einordnung der Wertschöpfungsstufen im Jahre 2004 dar. Kursive Schrift kennzeichnet 2008 im Vergleich zu 2004 neu hinzugekommene Aktivitäten. Die einzelnen Wertschöpfungsteilaktivitäten sind durch Kanten mit der jeweiligen Wertschöpfungsstufe, zu der sie zählen, verbunden.

\section{$5 \quad$ Fazit und Ausblick}

Ziel des vorliegenden Beitrags war es, zu untersuchen, wie sich die unternehmensexterne Arbeitsteilung und die daraus resultierende Leistungstiefe bei einem Unternehmen mit Open-Source-Geschäftsmodell entwickelt haben und damit die Studie eines Softwareunternehmens mit klassischem Closed-SourceGeschäftsmodell (SAP) von Wolf et al. (2008) zu ergänzen. Um zu beantworten, wie die unternehmensexterne Arbeitsteilung aus der Sicht eines typischen Softwareunternehmens mit Open-Source-Geschäftsmodell aktuell ausgeprägt ist, wie sie bei der Gründung des Unternehmens ausgestaltet war und bei welchen Wertschöpfungsaktivitäten sich Veränderungen ergeben haben, führten wir eine Fallstudienuntersuchung bei db4obejcts durch. 




Abbildung 3: Vergleich der unternehmensexternen Arbeitsteilung 2004 und 2008

Es zeigt sich, dass die unternehmensexterne Arbeitsteilung - ähnlich wie bei SAP in der Gründungszeit des Unternehmens recht niedrig ausgeprägt war und db4obejcts somit eine hohe Leistungstiefe aufwies. Genauso wie im zitierten Fall von SAP sank die Leistungstiefe dann über eine vergleichsweise kurze Zeit (2004-2009) und immer mehr Wertschöpfungsaktivitäten wurden unternehmensextern arbeitsteilig durchgeführt. Im Fall des Closed-Source-Geschäftsmodells von SAP wurden vor allem die Wertschöpfungsstufen des Dienstleistungsgeschäfts (Beratung, Implementierung, Schulung, Wartung) an Partnerunternehmen ausgelagert, um das immense Wachstum bewältigen zu können, während die Forschung und auch die Kernproduktentwicklung als Kernkompetenzen bei SAP selbst verblieben (Wolf et al. 2008, S. 167). Im Fall des Open-Source-Geschäftsmodells von db4obejcts wurde hingegen vor allem Teilaktivitäten der Wertschöpfungsstufe Produktentwicklung (Design, Produkt-Roadmap, Erstellung von Plugins und Addons, Qualitätsmanagement, Wartung des Kernprodukts) im Sinne einer interaktiven Wertschöpfung 
an die Nutzer-Community ausgelagert. Dies war zur Gründungszeit noch nicht möglich, da durch die zu Beginn geringen Nutzerzahlen und die nicht vorhanden User-Experience noch keine Community rund um das Produkt vorhanden war. Insbesondere die Wertschöpfungsstufe des Vertriebs verblieb hingegen bei db4objects. Es erscheint nachvollziehbar, dass der kommerzielle Vertrieb - auch eines Open-Source-Produkts - nicht von einer Entwickler-Community, sondern nur von einem klassischen Unternehmen mit Gewinnerzielungsabsicht erfolgreich übernommen werden kann.

Ein wie aktuell bei db4objects vorliegendes Wertschöpfungssystem, in dem eine Vielzahl von Akteuren gemeinsam Leistungen erbringen, wird bei traditionellen Unternehmen als Unternehmensnetzwerk bezeichnet (Hensel 2007, S. 23-24; Sydow 1993, S. 79). Bei Open-Source-Software handelt es sich allerdings insbesondere im Bereich der Entwicklung nicht um Unternehmen, die miteinander kooperieren, sondern um Anwender aus der Community, die untereinander und mit dem Projekt-Initiator (hier db4objects) - in den meisten Fällen ohne expliziten Kooperationsvertrag - zusammenarbeiten.

Zusammenfassend zeigt sich bei dem hier betrachteten Fall grundsätzlich auch eine Zunahme der unternehmensexternen Arbeitsteilung. Diese erfolgt interessanterweise jedoch im Gegensatz zum Closed-Source-Geschäftsmodell von SAP hauptsächlich in Teilbereichen der Entwicklung, was mit dem speziellen Community-Charakter der Erstellung von Open-Source-Software begründet werden kann.

Einschränkungen bei der Verallgemeinerung unserer Ergebnisse ergeben sich durch die bekannten Grenzen der qualitativen Betrachtung eines einzelnen Falles, die zwar eine hohe interne, gleichzeitig jedoch eine eingeschränkte externe Validität zur Folge hat. Daher sollte zukünftige Forschung in diesem Bereich unsere Ergebnisse einer quantitativen Kontrolle unterziehen und darauf aufbauend überprüfen, ob die festgestellten Entwicklungen der unternehmensexternen Arbeitsteilung im Spezialfall Open-Source mit den Vorhersagen etablierter Theorien wie der Transaktions- oder Produktionstheorie in Einklang stehen.

\section{Literatur}

Buxmann P, Diefenbach H, Hess T (2008) Die Softwareindustrie: Ökonomische Prinzipien, Strategien, Perspektiven. Springer, Berlin.

Fauser B (2004) Horizontale und vertikale Integration im Bereich der Leistungsverwertung. Hampp, München.

Franz A (2003) Management von Business Webs. DUV, Wiesbaden.

Hensel J (2007): Netzwerkmanagement in der Automobilindustrie: Erfolgsfaktoren und Gestaltungsfelder. Gabler, Wiesbaden.

Kretschmer W (2005) Die Neuen Stärken. Brand Eins7(4): 80-86. 
Laux H, Liermann F (1993) Grundlagen der Organisation: Die Steuerung von Entscheidungen als Grundproblem der Betriebswirtschaft. Springer, Berlin.

Meyer JA (2003) Die Fallstudie in der betriebswirtschaftlichen Forschung und Lehre. WiSt 32(8): 475-479.

Picot A (1991) Ein neuer Ansatz zur Gestaltung der Leistungstiefe. ZfbF 43(4): 336-357.

Reichwald R, Piller F (2006) Interaktive Wertschöpfung: Open Innovation, Individualisierung und neue Formen der Arbeitsteilung. Gabler, Wiesbaden.

Remer A (1996) Organisationslehre. REA, Bayreuth.

Richter C (2003) Einfluss organisationaler Steuerbarkeit auf Des-/Integrationsentscheidungen von Unternehmen: Entwicklung eines heuristischen Bezugsrah-mens und seine Überprüfung anhand der Branchen Automobil und Finanzdienstleister. Hampp, München.

Sydow J (1993) Strategische Netzwerke: Evolution und Organisation.

Wiederabdruck von 1992. Gabler, Wiesbaden.

Yin RK (2003) Case study research - design and method. Sage, Thousand Oaks.

Sharma S, Sugumaran V, Rajagopalan B (2002) A framework for creating hybrid open source software communities. Inf. Syst. Journal 12(1):7-25.

Williamson OE (1991) Comparative economic organization: the analysis of discrete structural alternatives. Administrative Science Quarterly 36(2): 269296.

Williamson OE (2002) The theory of the firm as governance structure: from choice to contract. Journal of Economic Perspectives 16(13): 171-195.

Wolf CM, Geiger K, Benlian A, Hess T, Buxmann P (2008) Spezialisierung als Ausprägungsform einer Industrialisierung der Software-Branche - Eine Analyse am Beispiel der ERP-Software von SAP. In Herzwurm G, Mikusz M (Hrsg) Industrialisierung des Software-Managements. GI, Stuttgart.

Zäpfel G (1989) Taktisches Produktions-Management. De Gruyter, Berlin 